\title{
2D Kagomé Ordering in the 3D Frustrated Spinel $\mathrm{Li}_{2} \mathrm{Mn}_{2} \mathrm{O}_{4}$
}

\author{
C. R. Wiebe, ${ }^{1,2, \circledast}$ P. L. Russo, ${ }^{1}$ A. T. Savici, ${ }^{1}$ Y. J. Uemura,${ }^{1}$ \\ G. J. MacDougall, ${ }^{2}$ G. M. Luke, ${ }^{2}$ S. Kuchta,${ }^{3}$ and J. E. Greedan ${ }^{3}$ \\ ${ }^{1}$ Department of Physics, Columbia University, New York, New York 10027, USA \\ ${ }^{2}$ Department of Physics and Astronomy, McMaster University, Hamilton, Ontario L8S 4M1, Canada \\ ${ }^{3}$ Department of Chemistry, McMaster University, Hamilton, Ontario L8S 4M1, Canada
}

(Dated: November 2, 2018)

\begin{abstract}
$\mu \mathrm{SR}$ experiments on the geometrically frustrated spinel oxide, $\mathrm{Li}_{2} \mathrm{Mn}_{2} \mathrm{O}_{4}$, show the development of spin correlations over a range of length scales with decreasing temperature. Increased relaxation below $\sim 150 \mathrm{~K}$ is consistent with the onset of spin correlations. Below $50 \mathrm{~K}$, spin order on a length scale, which is long range for the $\mu \mathrm{SR}$ probe, appears abruptly in temperature, consistent with prior neutron diffraction results. The oscillations in the zero field asymmetry are analyzed using a three frequency model. By locating the muon site this is shown to be consistent with the unexpected $2 \mathrm{D} \mathrm{q}=\sqrt{3} \times \sqrt{3}$ structure on the Kagomé planes proposed originally from neutron data. Longitudinal field data demonstrate that some spin dynamics persist even at $2 \mathrm{~K}$. Thus, a very complex magnetic ground state, featuring the co-existence of long length scale $2 \mathrm{D}$ ordering and significant spin dynamics, is proposed. This is unusual considering the $3 \mathrm{D}$ topology of the $\mathrm{Mn}^{3+}$ spins in this material.

PACS numbers: 75.10.-b, 76.75.+i, 75.40.-s
\end{abstract}

\section{INTRODUCTION}

The phenomenon of geometric frustration is now seeing a surge of interest due to the growing number of unique ground states which arise from networks of spins in triangular motifs. 1], 2] In particular, over the last few years, discoveries such as heavy-fermion behavior, [3] spin-ice ordering, 4] and even novel superconductivity [5] have been observed in materials which have magnetic sublattices of corner-shared tetrahedra (such as the spinels and pyrochlores). This has led to increased theoretical interest in these systems, which were originally suggested by Anderson to be excellent candidates for exotic magnetism, such as the dynamic resonating valence bond state. [6] Although very few examples in the literature exist of this elusive class of materials, new discoveries of the complex magnetism in these systems continue to intrigue the condensed matter community.

One of the more curious discoveries of late lies within the spinel materials $\mathrm{Li}_{1+x} \mathrm{Mn}_{2} \mathrm{O}_{4}$. These were once targets for the lithium ion battery community, but they were later discovered to have complicated magnetic properties based upon the frustrated sublattice that they have in common. Complete removal of $\mathrm{Li}$ species results in $\lambda$ $\mathrm{MnO}_{2}$, which orders below $32 \mathrm{~K}$ into a magnetic unit cell with 256 spins. 7] The next member in the series, $\mathrm{LiMn}_{2} \mathrm{O}_{4}$, exhibits partial $\mathrm{Mn}^{3+} / \mathrm{Mn}^{4+}$ charge ordering at $280 \mathrm{~K}$ followed by a very complex 3D long range magnetic order below $60 \mathrm{~K}$. Remarkably, the long range order co-exists with short range, nearest neighbor length scale, order down to the lowest temperature investigated, 1.5 K. [8], 20] But it is the material $\mathrm{Li}_{2} \mathrm{Mn}_{2} \mathrm{O}_{4}[9]$ which has perhaps the most remarkable ground state. This material is synthesized by chemie douce, or soft chemistry, insertion of $\mathrm{Li}$ ions into $\mathrm{LiMn}_{2} \mathrm{O}_{4}$. The magnetic sublattice, which is populated by $\mathrm{Mn}^{3+}$ spins, has a slight tetragonal distortion (due to the Jahn-Teller effect) from the ideal corner-shared tetrahedral network that is seen in the cubic pyrochlores. Nonetheless, this distortion is small, and the sublattice remains, topologically, three dimensional (see figure 1). D.C. magnetic susceptibility data showed signs of short range correlations up to $\mathrm{T} \sim$ $400 \mathrm{~K}$. Neutron diffraction on powder samples revealed broad features indicative of short range magnetic correlations appearing below $150 \mathrm{~K}$. With decreasing temperature these features took on a pronounced Warren line shape, a signature of $2 \mathrm{D}$ correlations. 10] This is a remarkable result given the 3D topology of the Mn sublattice. The intensity of the Warren reflections increased sharply between $50 \mathrm{~K}$ and $40 \mathrm{~K}$ in an almost first-order fashion. The maximum 2D spin correlation length, obtained by fitting to the Warren function, was $\sim 90 \AA$, a relatively large but finite value, which remained unchanged from $40 \mathrm{~K}$ to $1.6 \mathrm{~K}$. From the positions of the magnetic reflections, the authors suggested that the $2 \mathrm{D}$ correlations could be assigned to a $\mathrm{q}=\sqrt{3} \times \sqrt{3}$ structure confined to the Kagomé sheets (see figure 1), but this assignment was based on the observation of only two magnetic reflections. [9]

In this paper, $\mu \mathrm{SR}$ experiments confirm the onset of a long ranged (on the $\mu \mathrm{SR}$ length scale) spin ordering below $50 \mathrm{~K}$, and show evidence for the very short range order setting in below $150 \mathrm{~K}$. Moreover, substantial evidence is provided for the $\mathrm{q}=\sqrt{3} \times \sqrt{3}$ magnetic structure on Kagomé layers proposed from the neutron data, and may suggest either a $2 \mathrm{D}$ order parameter, or the first-order nature of the transition. 
(a)

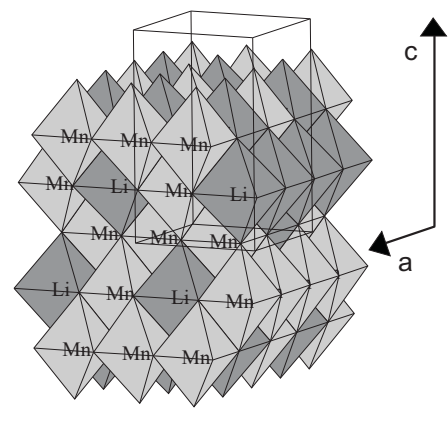

(b)

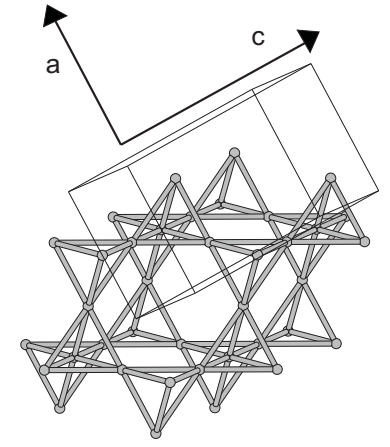

FIG. 1: (a) A polyhedral representation of the structure of $\mathrm{Li}_{2} \mathrm{Mn}_{2} \mathrm{O}_{4}$ showing the $\mathrm{Li}-\mathrm{O}$ and $\mathrm{Mn}-\mathrm{O}$ octahedra. The $\mathrm{Li}$ ions have migrated from the tetrahedral sites in the normal spinel, $\mathrm{LiMn}_{2} \mathrm{O}_{4}$, to the octahedral sites in $\mathrm{Li}_{2} \mathrm{Mn}_{2} \mathrm{O}_{4}$. (b) The Mn magnetic sublattice, shown as a network of cornershared tetrahedra (which would be identical to the cubic pyrochlore sublattice if not for the tetragonal distortion). This can be described as Kagomé layers which alternate with triangular planar layers in the stacking direction normal to the $<111>$ direction.

\section{EXPERIMENTAL PROCEDURE AND RESULTS}

\section{Sample Preparation and Characterization}

$\mathrm{Li}_{2} \mathrm{Mn}_{2} \mathrm{O}_{4}$ was synthesized according to the method of Wills et al. by chemie douce, or soft chemistry insertion of $\mathrm{Li}$ ions into $\mathrm{LiMn}_{2} \mathrm{O}_{4}$. $\mathrm{LiMn}_{2} \mathrm{O}_{4}$ was prepared by reacting stoichiometric amounts of $\mathrm{Li}_{2} \mathrm{CO}_{3}$ and $\mathrm{Mn}_{2} \mathrm{O}_{3}$ in air at $650^{\circ} \mathrm{C}$ for 12 hours and $800^{\circ} \mathrm{C}$ for 24 hours, followed by a gradual cooling to room temperature. The product was reground and refired according to the same heating routine and then tested for phase purity by x-ray diffraction using $\mathrm{K}_{\alpha 1}$ radiation on a Bruker $\mathrm{D} 8$ diffractometer. The $\mathrm{Li}$ insertion step was completed in an $\mathrm{Ar}$ glove box. Three grams of finely ground $\mathrm{LiMn}_{2} \mathrm{O}_{4}$ were added to $12.5 \mathrm{~mL}$ of $16 \mathrm{M} \mathrm{n}$-butyl lithium in $40 \mathrm{~mL}$ of sodium-dried hexane. This provides a slight excess of $n$-butyl lithium. After gentle heating at $40^{\circ} \mathrm{C}$ for 5 days, the final product was filtered and washed well with sodium-dried hexane. The product was tested for phase purity using a Guinier-Hägg camera in a sealed mylar sample holder.

The magnetic susceptibility was measured in a sealed capsule using a Quantum Design SQUID magnetometer. The experiments were completed in field-cooled (FC) and zero field-cooled (ZFC) sequences and using the reciprocating sample option (RSO) mode on the instrument. $\mu \mathrm{SR}$ experiments were completed by enclosing the sample in a mylar-sealed sample holder with $\mathrm{He}$ exchange gas. All experiments were completed at the M20 beamline at TRIUMF, Vancouver, Canada in a He-flow cryo-

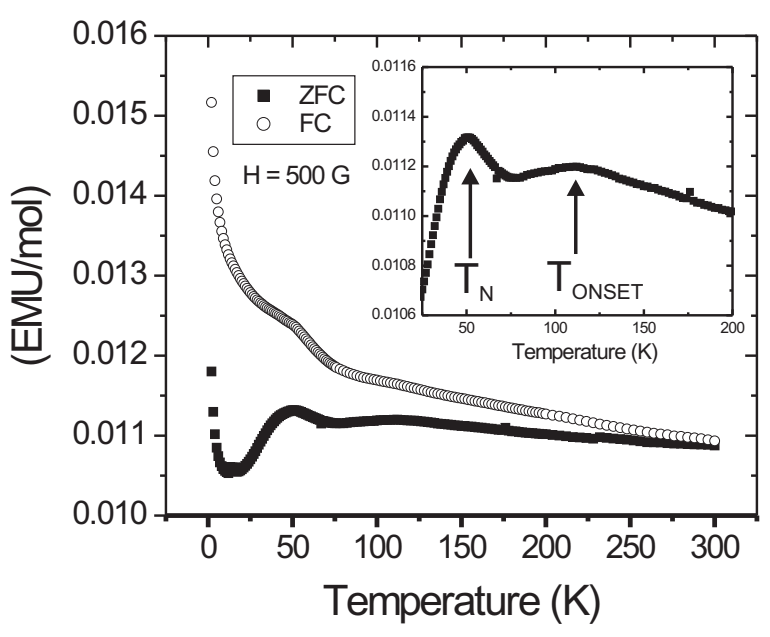

FIG. 2: The DC magnetic susceptibility of $\mathrm{Li}_{2} \mathrm{Mn}_{2} \mathrm{O}_{4}$ for an applied field of $\mathrm{H}=500 \mathrm{G}$. Zero field-cooled (ZFC) and fieldcooled data sets $(\mathrm{FC})$ are denoted. A broad feature at $\sim 110$ $\mathrm{K}$ suggests the onset of magnetic correlations ( $\mathrm{T}_{\text {ONSET }}$ ), and a sharper peak at $\sim 50 \mathrm{~K}$ corresponds to the Néel temperature $\left(\mathrm{T}_{N}\right)$. The FC/ZFC divergence at higher temperatures is due to magnetic correlations (there is no evidence for impurities through x-ray diffraction measurements).

stat which reached temperatures from $2 \mathrm{~K}$ to $150 \mathrm{~K}$. Zerofield (ZF) and longitudinal field (LF) measurements were made in applied fields of up to $2 \mathrm{kG}$.

\section{Experimental Results}

DC magnetic susceptibility measurements made in an applied field of $500 \mathrm{G}$ qualitatively reproduced the previous results of Wills and co-workers (see figure 2). There was no Curie-Weiss behavior detected up to $350 \mathrm{~K}$, and indeed, previous measurements detected no such behavior up to $800 \mathrm{~K} .[9]$ This is a signature of frustrated systems - the Curie-Weiss region is pushed to higher temperatures due to strong antiferromagnetic interactions between the spins. There is also a broad feature at $\mathrm{T} \sim$ $110 \mathrm{~K}$ which makes it difficult to perform a Curie-Weiss analysis. This peak is interpreted as a signature of shortranged magnetic ordering of the $\mathrm{Mn}^{3+}$ spins. A cusp in the susceptibility occurs just below $50 \mathrm{~K}$, indicative of the onset of the relatively long range $2 \mathrm{D}$ order found in the neutron data. The divergence of the FC/ZFC data at high temperatures could be due to a small impurity, or the presence of magnetic correlations. The lack of impurity lines in the x-ray diffraction data suggests the latter explanation over the former.

$\mu \mathrm{SR}$ measurements in zero-field (ZF) identified a two- 
component lineshape which became prominent below 150 $\mathrm{K}$. This data was fit to a Kubo-Toyabe function, to account for relaxation processes from nuclear dipoles, and an exponential function, to account for relaxation of magnetic origin.

$$
P(t)=A\left(f G_{K T} e^{-\lambda_{\text {slow }} t}+(1-f) e^{-\lambda_{\text {fast }} t}\right)
$$

where $\mathrm{P}(\mathrm{t})$ is the muon polarization function, $\mathrm{A}$ is the asymmetry, $f$ is the nuclear dipolar fraction, $G_{K T}$ is the Kubo-Toyabe lineshape, and $\lambda_{\text {slow }}$ and $\lambda_{\text {fast }}$ are the relaxation rates (see figure 3 ). The former rate is slower than the latter, which accounts for the quasi-static ordering seen above $50 \mathrm{~K}$ as a decrease in polarization at early times. The fraction, $\mathrm{f}$, represents the fraction of the slow Kubo-Toyabe signal of the total signal. At high temperatures (ie. $150 \mathrm{~K}$ ), this is 1 . As one cools down (see figure $5(\mathrm{a})$ ), this fraction decreases as the fast exponential component grows in size. This shows that a separate population of spins starts to slow down and grow in size below $150 \mathrm{~K}$, which correlates nicely with the broad feature in the DC susceptibility at $110 \mathrm{~K}$. At $57 \mathrm{~K}$, this population represents about 25 percent of the total asymmetry. Below $50 \mathrm{~K}$, about a third of the asymmetry is oscillating (corresponding to about 50 percent of the spins ordered).

The Kubo-Toyabe lineshape is, explicitly:

$$
G_{K T}=\frac{1}{3}+\frac{2}{3}\left(1-\Delta^{2} t^{2}\right) e^{-\Delta^{2} t^{2} / 2}
$$

where $\Delta^{2}$ is the second moment of the field distribution of nuclear dipolar origin. A rise in the relaxation rate to a maximum at $50 \mathrm{~K}$ is typical of the dynamics associated with magnetic transitions to long range ordered ground states. The physical origin of this is the longer correlation times near a phase transition (known as critical slowing down). The lack of a sharp maximum at $50 \mathrm{~K}$ could be due to either the coarseness of the temperature steps near $\mathrm{T}_{N}$, or the abrupt nature of the transition. The appearance of a second component at temperatures much larger than $50 \mathrm{~K}$, however, is unusual and its origin is due to the gradual slowing down of spins over decades in temperature (another common feature of magnetically frustrated systems). A likely cause of this is the formation of short-ranged spin correlations, as suggested by previous neutron work. It is also unusual that the relaxation persists to low temperatures below $50 \mathrm{~K}$. This is evidence for fluctuating spins co-existing with ordered spins as $\mathrm{T} \rightarrow 0 \mathrm{~K}$. Further signatures of the dynamic nature of this ground state is seen in the longitudinal field measurements (LF), which show that even strong fields do not completely decouple the signal.

$\mathrm{ZF}-\mu \mathrm{SR}$ spectra taken below $50 \mathrm{~K}$ show an oscillating component from muon precession in a local internal magnetic field. Fourier transforms revealed three well-defined (a)

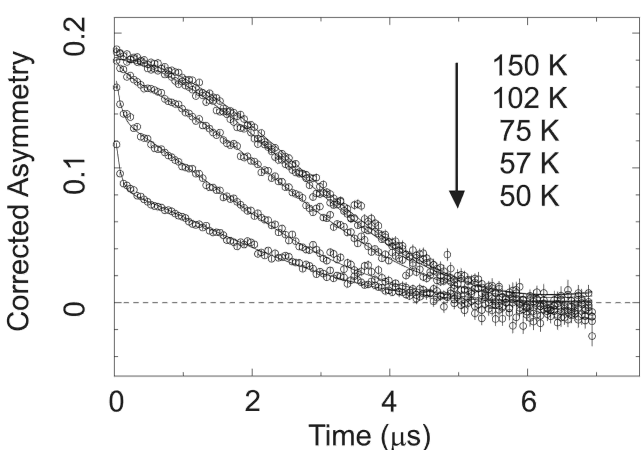

(b)

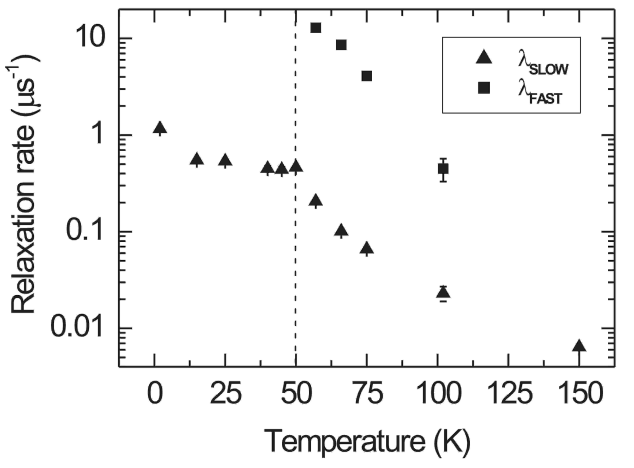

FIG. 3: (a) ZF- $\mu$ SR spectra at $50 \mathrm{~K}$ and above with fits described in the text. (b) Relaxation rate of the the two components as a function of temperature. Below $50 \mathrm{~K}$, a different fitting function is used for the data.

frequencies, which were used to fit the data below $50 \mathrm{~K}$ in the regime $0 \mathrm{~s}<\mathrm{t}<2 \mu \mathrm{s}$ (see figure 4). For longer time scales, the data was fit to a Kubo-Toyabe lineshape multiplied by an exponential.

$$
\begin{array}{r}
P(t)=A G_{K T} e^{-\lambda_{\text {slow }} t}+A_{1} \cos \left(\omega_{1} t\right) e^{-\lambda_{1} t} \\
+A_{2} \cos \left(\omega_{2} t\right) e^{-\lambda_{2} t}+A_{3} \cos \left(\omega_{3} t\right) e^{-\lambda_{3} t}
\end{array}
$$

In this equation, $\mathrm{A}_{i}, \lambda_{i}$, and $\nu_{i}$ are the asymmetries, relaxation rates, and frequencies of the three components, respectively. A common phase, $\phi$, was used for the three components for the initial fitting process. Since this was within error of zero, the phase was set to zero for the final fits.

The asymmetries of the oscillating and non-oscillating components are noted in figure 5. The drop in asymmetry of the ordered signal at $50 \mathrm{~K}$ coincides with a rise of the Kubo-Toyabe/exponential signal.

The asymmetry of the oscillating components was found to be only about a third of the expected total asymmetry, suggesting that only a portion of the spins are ordered at $2 \mathrm{~K}$. The remainder of the spins are either still fluctuating or in short-ranged ordered spin clusters. The three frequency fit represents the data well, however the corresponding temperature dependence of all three 
(a)
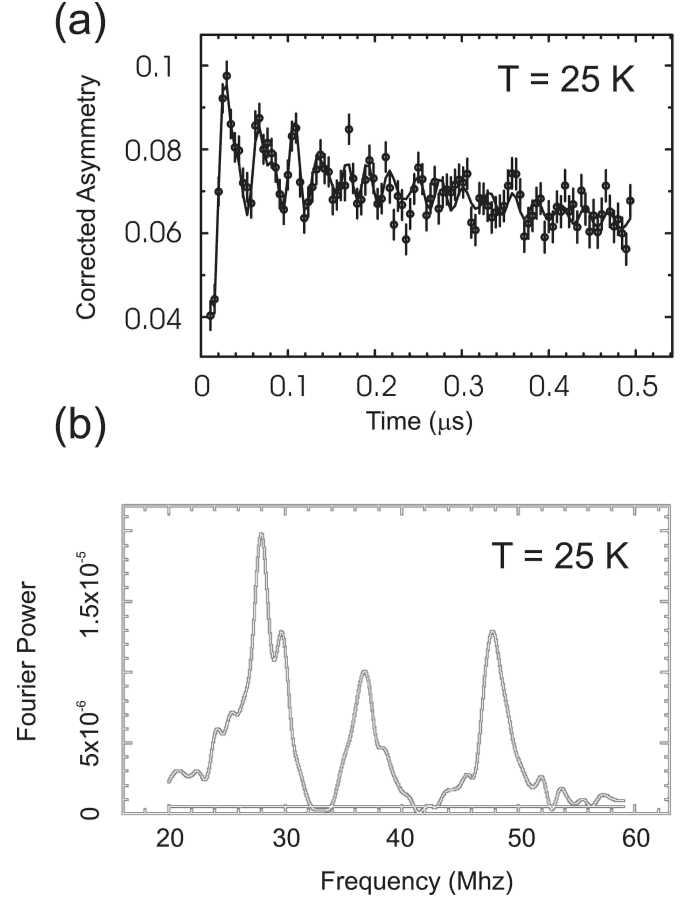

FIG. 4: (a) Early time ZF- $\mu$ SR spectra at $\mathrm{T}=25 \mathrm{~K}$. The fit is to a three frequency model. (b) The Fourier transform of this data, showing three distinguishable components.

components is uncharacteristic of $3 \mathrm{D}$ ordering. All three show a very rapid increase to saturation below $50 \mathrm{~K}$.

The low frequency component of the data has been fit to the phenomenological form:

$$
\nu=\nu_{0}\left(1-T / T_{N}\right)^{\beta}
$$

where $\beta$ is the critical exponent. Although a rigorous examination of the power law would require more points around $\mathrm{T}_{N}$, it is clear that the transition is not mean-field like $(\beta=0.5)$, or $3 \mathrm{D}$ Heisenburg $(\beta=0.33)$, as shown in figure 6 . The fit, to free parameters for $\beta, \nu_{0}$, and $\mathrm{T}_{N}$ for the lowest frequency component, is $\beta=0.14(3)$, which is more consistent with the $2 \mathrm{D}$ Ising critical exponent of $\beta=0.125$, and a transition temperature of $48+/-$ $2 \mathrm{~K}$. The rapid increase of the precession frequency is also characteristic of a first-order like transition, which is echoed in the spin-spin correlation lengths extracted from neutron scattering measurements.

The relaxation rate of the $1 / T_{1}$ tail is approximately a constant below $\mathrm{T}_{N}$. This is strong evidence that there are coexisting regions of ordered and disordered spins. Since the behaviour of the disordered spins (from the $1 / \mathrm{T}_{1}$ tail) changes abruptly below $\mathrm{T}_{N}$, these spins must be involved with the transition (it is unlikely that there is bulk phase separation). (a)

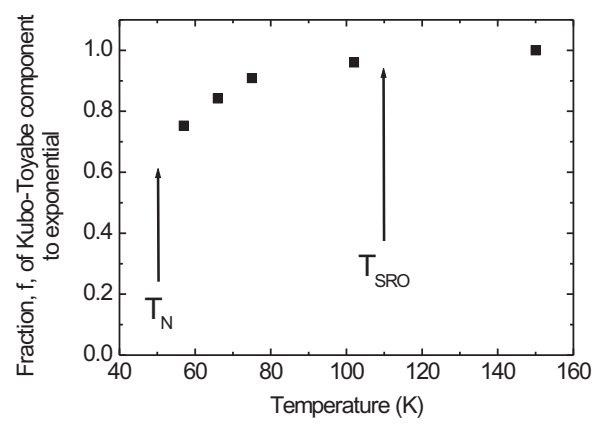

(b)

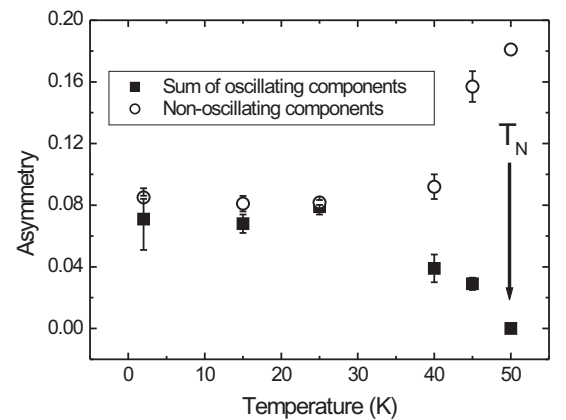

FIG. 5: (a) The temperature dependence of the Kubo-Toyabe signal to the exponential signal above $50 \mathrm{~K}$. (b) The temperature dependence of the three oscillating components (summed together) and the non-oscillating Kubo-Toyabe function (multiplied by an exponential).

LF- $\mu$ SR spectra show that most of the signal is decoupled with the application of applied magnetic fields. This indicates that there is a large population of static spins. However, there is a small fraction of spins (about 10 percent of the total volume fraction) which do not decouple, even down to $2 \mathrm{~K}$ and in strong fields (see figure 7 at $\mathrm{T}=2 \mathrm{~K}$ and $\mathrm{T}=66 \mathrm{~K}$ ). This suggests the coexistence of magnetic order and regions of persistently fluctuating moments.

\section{DISCUSSION}

Our $\mu \mathrm{SR}$ results present strong evidence for the model proposed by [9], i.e. a $\mathrm{q}=\sqrt{3} \times \sqrt{3}$ ordering for the Mn spins in the Kagomé layers. Below $50 \mathrm{~K}$, there appears to be a population of spins (roughly 50 percent) which lie in an ordered state with respect to the muon timescale. In order to shed some light on the nature of this ordering, one must first attempt to identify the muon site. Given that there are three separate frequencies in the data, the first place to start is to assume that the muon is experiencing three distinct internal fields from either the magnetic structure or different muon sites or 


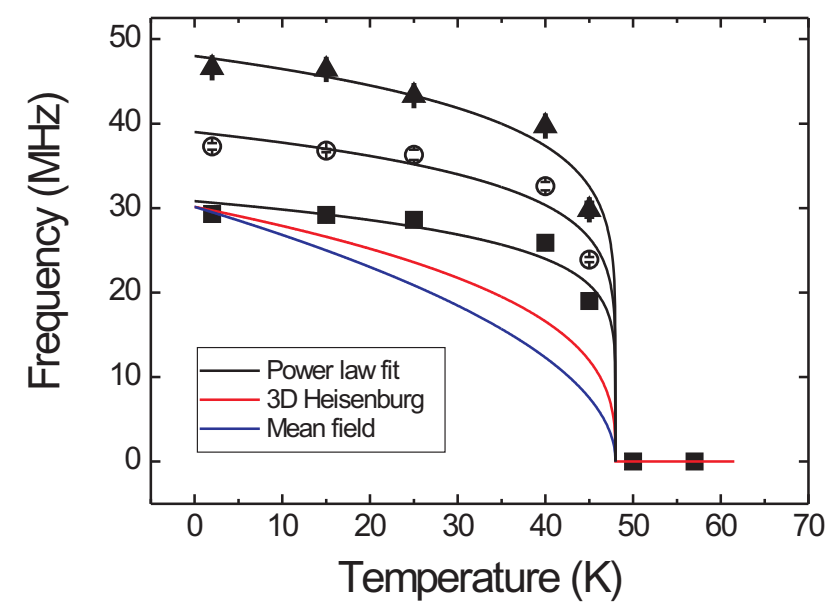

FIG. 6: (Colour online) The temperature dependence of the three fitted frequencies. A power-law exponential has been fitted to the lowest frequency with $\beta=0.14(3)$. The two higher frequencies show the same fit for the lower frequency with a normalization factor. For comparison, the expected power-laws for 3D Heisenburg and mean field theory have been shown.

both. It is therefore important to locate the muon sites within $\mathrm{Li}_{2} \mathrm{Mn}_{2} \mathrm{O}_{4}$. The most probable site is one which is equidistant from the oxygen positions. There are three obvious choices for this: at the interstitial sites $(3 / 8,3 / 8$, $3 / 8),(1 / 4,1 / 4,1 / 4)$, or $(0,0.75,0.125)$. Detailed calculations of the field experienced by the muon at each site were made by using the equation:

$$
\Delta^{2}=\frac{4}{9} \gamma_{\mu}^{2} \sum \overline{\mu_{i}^{2}} \frac{1}{r_{i}^{6}}
$$

in which $\gamma_{\mu}$ is the muon gyromagnetic ratio (135.5 $\mathrm{MHz} / \mathrm{T})$ and $\mu_{i}$ is the dipolar moment at site $i$ at a distance $r_{i}$ from the muon site. This is the relaxation rate $\Delta$ (due to random nuclear dipolar fields) which is extracted from the Kubo-Toyabe fits to the high temperature data $(150 \mathrm{~K})$. Sums were made over several unit cells in length in the three crystallographic directions. The theoretical values for the relaxation rates at the three sites of interest are listed in table I.

In the material $\mathrm{LiMn}_{2} \mathrm{O}_{4}$, the most probable site is in the interstitial position $(1 / 4,1 / 4,3 / 4)$, where the muon has some mobility within the lithium-depleted structure acting like $\mathrm{a} \mathrm{Li}^{+}$ion. This has been verified by detailed $\mu \mathrm{SR}$ experiments and calculations on $\mathrm{LiMn}_{2} \mathrm{O}_{4} \cdot 11$. However, in $\mathrm{Li}_{2} \mathrm{Mn}_{2} \mathrm{O}_{4}$, all of these sites are filled by intercalated lithium atoms. The calculated relaxation rate of $0.132 \mu \mathrm{s}^{-1}$ does not agree with the value found from the (a)

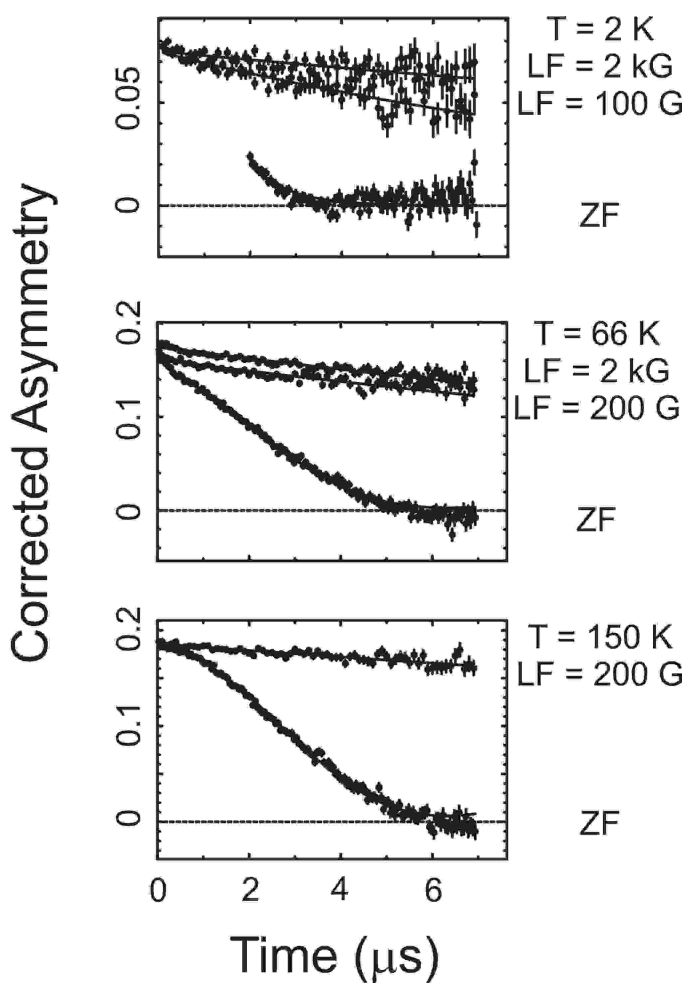

FIG. 7: $\mathrm{ZF}$ and $\mathrm{LF} \mu \mathrm{SR}$ data at (a) $\mathrm{T}=2 \mathrm{~K}$, (b) $\mathrm{T}=66 \mathrm{~K}$, (c) $\mathrm{T}=150 \mathrm{~K}$. The data at $2 \mathrm{~K}$ in $\mathrm{ZF}$ has been omitted in the early time signal where oscillations are observed.

\begin{tabular}{|c|c|c|}
\hline Muon site & $\Delta\left(\mu \mathrm{s}^{-1}\right)$ & Comment \\
\hline$(1 / 4,1 / 4,3 / 4)$ & 0.132 & Occupied by Li \\
$(3 / 8,3 / 8,3 / 8)$ & 0.418 & Interstitial \\
$(0,0.75,0.125)$ & 0.308 & Interstitial \\
\hline
\end{tabular}

TABLE I: Possible muon sites with the calculated relaxation rate. The $(0,0.75,0.125)$ position has a value which is closest to the fits derived from the muon data $\left(\Delta=0.275 \mu \mathrm{s}^{-1}\right)$.

Kubo-Toyabe fits of $0.275 \mu \mathrm{s}^{-1}$, suggesting that another muon site is a better choice.

The site $(3 / 8,3 / 8,3 / 8)$ is in an electrostatically favorable environment, surrounded by 6 oxygens in an octahedral framework at distances of 1.5 to $2.6 \AA$. This position was found to be the muon site for the spinel $\mathrm{LiV}_{2} \mathrm{O}_{4}$ in a recent $\mu \mathrm{SR}$ study. 12. However, the theoretical value for the relaxation rate from this field distribution is 0.418 $\mu \mathrm{s}^{-1}$ which does not agree with the value obtained from our data.

The best agreement with our high temperature KuboToyabe fits to the relaxation rate of $0.275(1) \mu \mathrm{s}^{-1}$ is at the $(0,0.75,0.125)$ position. It is perhaps not surprising that this is the most probable muon stopping site for 
(a)

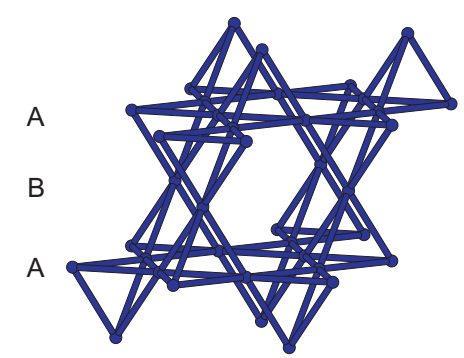

(b)

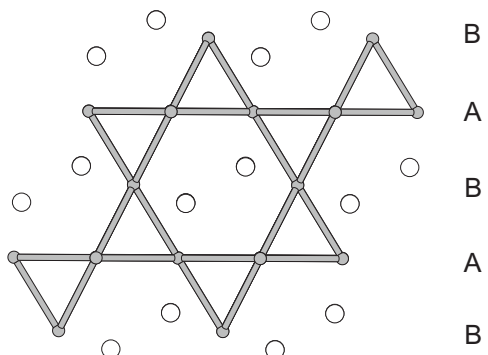

FIG. 8: (a) The Kagomé magnetic sublattice within the spinel structure. A and B indicate the alternating Kagomé and triangular planar layer sites, respectively. (b) The muon stopping sites with respect to the Kagomé (A) and interconnecting layers (B). (a)

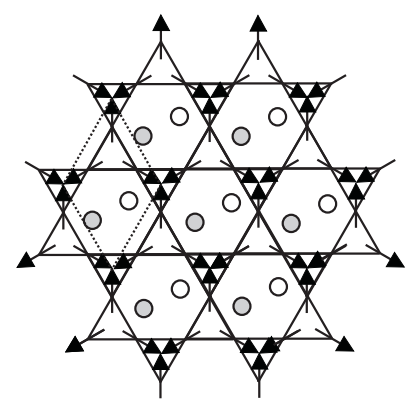

(b)

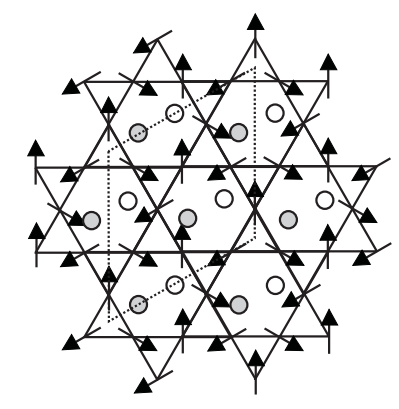

FIG. 9: (a) The $\mathrm{q}=0$ Kagomé structure and (b) the $\mathrm{q}=$ $\sqrt{3} \times \sqrt{3}$ structure. The muon sites in $\mathrm{Li}_{2} \mathrm{Mn}_{2} \mathrm{O}_{4}$ are indicated on the figures as circles, slightly above (grey) and below (white) the Kagomé planes. The magnetic sublattices in 2D are marked by dashed lines. There are two muon sites for the $q=0$ structure, but six sites for the $q=\sqrt{3} \times \sqrt{3}$ structure.

$\mathrm{Li}_{2} \mathrm{Mn}_{2} \mathrm{O}_{4}$. This is a tetrahedral site where Li resides in $\mathrm{LiMn}_{2} \mathrm{O}_{4}$, but it is not occupied in $\mathrm{Li}_{2} \mathrm{Mn}_{2} \mathrm{O}_{4}$ according to previous diffraction studies (all of the lithium atoms are at the octahedrally coordinated $8 c$ site $(0,0,0))$. This is, therefore, a favorable position for the muon, which will act like a $\mathrm{Li}^{+}$cation surrounded by a four-fold oxygen cage at distances of $1.92 \AA$. It is also surrounded by 4 equidistant $\mathrm{Li}^{+}$cations at distances of $1.82 \AA$.

Given this result, one can now shed some light on the muon precession frequencies observed at the ordering transition. Wills and co-workers suggested that a $\mathrm{q}$ $=\sqrt{3} \times \sqrt{3}$ Kagomé ordering is present within the pyrochlore Mn magnetic sublattice. This leaves two separate magnetic species - the ordered spins which lie within the Kagomé layers and the disordered spins in the adjacent triangular plane layers. Figure 8 shows this explicitly, with the muon stopping sites indicated. These are located slightly above and below the Kagomé planes, at positions roughly in the middle of each Kagomé plaquette. Three different muon precession frequencies are seen for our data, at roughly $46 \mathrm{MHz}, 37 \mathrm{MHz}$, and $30 \mathrm{MHz}$, corresponding to three different internal fields seen by the muon. To see how this could arise in the $\mathrm{q}=\sqrt{3} \times \sqrt{3}$ spin structure, consider figure 9 . The two possible spin structures are plotted with respect to the muon stopping sites, which lie at positions just above and below the planes as indicated. For the $\mathrm{q}=0$ structure, the muons all experience virtually the same spin environment within the magnetic unit cell. In the upper limit, the muon can feel two frequencies within the unit cell (one for each stopping site). However, there are six different internal fields experienced by muons within the $\mathrm{q}=\sqrt{3} \times \sqrt{3}$ magnetic unit cell. Detailed calculations of the internal fields using dipolar interactions between the moments and the muon sites reveal that there are indeed three frequencies experienced by the muons - 4 sites which are nearly degenerate with a low frequency, and the remaining two sites with higher frequencies. Table II shows these results explicitly, with excellent agreement shown with the ratios of the three frequencies observed in our data. This also is in qualitative agreement with the distribution of components in the fourier transform, which reveals a broad set of frequencies centered about $30 \mathrm{MHz}$ (from the four nearly degenerate fields), and two smaller components at 37 and $46 \mathrm{MHz}$ (from the other two fields observed). In conclusion, one can say with some confidence that the $\mathrm{q}$ $=\sqrt{3} \times \sqrt{3}$ structure is consistent with the distribution of frequencies observed in our data, given our choice of muon site. The $\mathrm{q}=0$ structure cannot give rise to three unique muon frequencies.

\begin{tabular}{|c|c|c|}
\hline Frequency (MHz) & Ratio (exp.) & Ratio (calc.) \\
\hline $29.3+/-0.4$ & - & - \\
$37.3+/-0.4$ & $1.27+/-0.04$ & 1.34 \\
$46.4+/-0.4$ & $1.58+/-0.04$ & 1.46 \\
\hline
\end{tabular}

TABLE II: Experimental frequency ratios for precession components at $2 \mathrm{~K}$. The ratios of these frequencies are compared to the calculated values for the muon site in the $\sqrt{3} \times \sqrt{3}$ structure.

With this excellent agreement of the internal field at the muon sites with respect to the ratios of the frequencies observed, one can now estimate the size of the average static moment at the $\mathrm{Mn}^{3+}$ site. This is done by evaluating the the size of the field at these points by dividing the precession frequency by the muon gyromagnetic ratio $\left(\gamma_{\mu}=135.5 \mathrm{MHz} / \mathrm{T}\right)$, and then comparing this to our 
calculations of the frequency distribution. The resultant average static moment of 1.5(1) $\mu_{B}$ per atom is shy of the expected value for $\mathrm{Mn}^{3+}$ moments of $4.0 \mu_{B}$. Reduced moments are common in magnetically frustrated systems. 2] Another possible origin of the small moment is that there are some disordered spins within the ordered Kagomé layers, leading to a lower average static moment.

In subsequent $\mathrm{LF}-\mu \mathrm{SR}$ experiments, we observed that applied fields do not completely decouple the signal (see figure 6), which suggests that the dynamics are not quenched easily. The rise of the relaxation rate to a constant value below $50 \mathrm{~K}$ is consistent with a dynamic ground state as well. Other frustrated systems, such as $\mathrm{Tb}_{2} \mathrm{Ti}_{2} \mathrm{O}_{7}$, show this behaviour, which has been interpreted as a ground state stabilized by quantum fluctuations. 13] The asymptotic relaxation rate, $\sim$ $1 \mu \mathrm{s}^{-1}$, is of the same order of magnitude. This distinguishes the behavior of this system from that associated with glassy spin freezing, such as seen in $\mathrm{Y}_{2} \mathrm{Mo}_{2} \mathrm{O}_{7}$, which has a prominent cusp in the relaxation rate and a low temperature value which is about 250 times smaller than $\mathrm{Li}_{2} \mathrm{Mn}_{2} \mathrm{O}_{4}$. 14] Highly correlated dynamics typical of frustrated materials, such as spin-liquid behavior, 15. would give rise to purely exponential relaxation, or "undecoupable" gaussian features in zero-field $\mu \mathrm{SR}$.16 $\mathrm{Li}_{2} \mathrm{Mn}_{2} \mathrm{O}_{4}$ is qualitatively different from either a pure spin glass or a pure spin liquid, with the co-existence of ordered and fluctuating spins.

This partial ordering phenomena is rare in magnetism, but perhaps not unexpected. Intercalated materials are often found to be multi-phasic, for example, by virtue of the reaction process, which depends on the reaction kinetics of butyl-lithium on micrograins of sample. This explanation seems unlikely, however, due to the lack of impurity lines found in x-ray powder diffraction data using the Guinier camera. Partial magnetic ordering at $\mathrm{T}_{N}$ has been seen in a wide variety of materials, and similar behavior has been seen in the $\mu \mathrm{SR}$ literature. One material in particular, the oxygen doped superconductor $\mathrm{La}_{2} \mathrm{CuO}_{4+\delta}$, has recently been examined by Savici et al. [17] It is believed, from the perspective of various experimental probes, that the magnetism develops with the superconductivity at the same temperature. However, the magnetic component develops in an abrupt way - that is, there is a rapid rise in the frequency of the muon signal at $T_{N}$ which is atypical of three dimensional phase transitions. This is not due to a poor quality of fit to the data - like in $\mathrm{Li}_{2} \mathrm{Mn}_{2} \mathrm{O}_{4}$, the frequency has a discontinuous evolution at $\mathrm{T}_{c}$. In $\mathrm{La}_{2} \mathrm{CuO}_{4+\delta}$, the explanation for this result is that there is a spin-density wave structure (as revealed by neutron scattering) in isolated, two dimensional "islands" of spins which reside within the superconducting fluid. The small asymmetry of the oscillating component (of about 30 percent with respect to the total asymmetry) was used to calculate the the size of these islands, and a corresponding "swiss-cheese" model of superconductivity was developed to explain the results. It may be that in $\mathrm{Li}_{2} \mathrm{Mn}_{2} \mathrm{O}_{4}$, similar two dimensional islands of the ordered phase coexist within a sea of quasi-static spins. This could be due to inhomogeneities, or due to some exotic ordering mechanism which favours domain formation of such a ground state. Further studies are clearly needed to distinguish the two possibilities.

With respect to other spinel oxides, $\mathrm{Li}_{2} \mathrm{Mn}_{2} \mathrm{O}_{4}$ has common features with materials such as $\mathrm{ZnFe}_{2} \mathrm{O}_{4}$. The low temperature ground state of $\mathrm{ZnFe}_{2} \mathrm{O}_{4}$ is an ordered antiferromagnet with a $\mathrm{k}=(0,0,0)$ wavevector at 10 K. 19. However, it has been known for some time that a short range ordered state exists at temperatures much higher than $\mathrm{T}_{N}$ - as high as $100 \mathrm{~K}$. Broad peaks appear in elastic neutron scattering experiments which persist to low temperatures. In addition, the $\mu \mathrm{SR}$ data on this material shows a two-component lineshape below $100 \mathrm{~K}$ in accordance with the slowing down of spins in a short range ordered state. With neutron and $\mu \mathrm{SR}$ studies combined, the consensus is that at $\mathrm{T}_{N}=10 \mathrm{~K}$, only about 30 percent of the volume fraction is long range ordered. The remaining fraction are in "superantiferromagnetic" clusters of short range ordered spins with sizes of about 30 $\AA$ (incidentally, this is in good agreement with the spinspin correlation length in the $\mathrm{Li}_{2} \mathrm{Mn}_{2} \mathrm{O}_{4}$ neutron scattering data of about $20 \AA$ at $100 \mathrm{~K}[9]$ ). The similarities between these two materials are considerable, both with short range ordered states at high temperatures, followed by a transition to a co-existing long range ordered state at low temperatures. The $\mu \mathrm{SR}$ data on $\mathrm{ZnFe}_{2} \mathrm{O}_{4}$ suggests there is some competition between these two states below $\mathrm{T}_{N}$, with the volume fraction of the LRO clusters becoming larger at the expense of the SRO clusters. This has not been observed in $\mathrm{Li}_{2} \mathrm{Mn}_{2} \mathrm{O}_{4}$. Larger fields are also needed to decouple the LF- $\mu$ SR data in $\mathrm{ZnFe}_{2} \mathrm{O}_{4}$, which suggests that significant dynamics play a role at low temperatures. The ground state is inhomogeneous in both cases, but notable differences exist between these two materials.

A closer relative to $\mathrm{Li}_{2} \mathrm{Mn}_{2} \mathrm{O}_{4}$ is the single-lithiated spinel $\mathrm{LiMn}_{2} \mathrm{O}_{4}$. As mentioned above, this material orders into a LRO state at $60 \mathrm{~K}$. The magnetic unit cell is enormously complicated, with a $\mathrm{k}=(1 / 2,1 / 2,1 / 4)$ structure and 1152 spins within. [8] A detailed magnetic structure is at this time unavailable. However, it is known that there is a SRO state which exists to high temperatures. Recently, spin polarized neutron scattering measurements revealed that above $\mathrm{T}_{N}=65 \mathrm{~K}$ the observable magnetic cross section is entirely due to SRO spins on a length scale of $\sim 3.5 \AA$ (second Mn neighbors). 20] As the temperature is reduced below $\mathrm{T}_{N}$, the fraction of the total magnetic cross section associated with LRO increases, reaching a maximum value of only 50 percent at $1.5 \mathrm{~K}$. Inelastic scattering shows that even at $1.5 \mathrm{~K}, \sim 20$ percent of the spins are still fluctuating. $\mathrm{LiMn}_{2} \mathrm{O}_{4}$ thus shows a complex magnetic ground state with co-existing 
3D LRO, SRO, and fluctuating spins. This is similar to the situation found for $\mathrm{Li}_{2} \mathrm{Mn}_{2} \mathrm{O}_{4}$ but the details are different as there is no true LRO in this material but only 2D SRO of significant, but finite, extent at the lowest temperatures.

In broader terms of comparison, $\mathrm{Li}_{2} \mathrm{Mn}_{2} \mathrm{O}_{4}$ is very distinct from other frustrated sublattices. $\mathrm{Gd}_{2} \mathrm{Ti}_{2} \mathrm{O}_{7}$ has been suggested to be an example of a system which has a Kagomé ordering on a pyrochlore lattice. 21] The mechanism is very different in this case, with dipolar interactions influencing the low-temperature physics of the $\mathrm{Gd}$ moments ordering. However, the real canonical examples of the 2D Kagomé lattice are the jarosites. For years, $\mathrm{SrCr}_{x} \mathrm{Ga}_{12-x} \mathrm{O}_{19}$ (SCGO) was considered to be an example of the unusual physics which develops in Kagomé systems, being a material which has exhibited spin-glass to spin-liquid characteristics depending on the Cr-site coverage and sample quality. [16], 18] However, this material, being of the magnetoplumbite structure, is better described as an array of bilayer Kagomé units rather than the pure structure. The jarosites, of general formula $\mathrm{AB}_{3}\left(\mathrm{SO}_{4}\right)_{2}(\mathrm{OH})_{6}$, with $\mathrm{B}$ being the magnetic species, represent the best physical realization of the Kagomé lattice. 222], 23] Although disorder within these layers is common, most of these materials have a high site coverage that is still within the percolation limit. A large portion of them form LRO ground states as well, in either the $\mathrm{q}=0$ or $\mathrm{q}=\sqrt{3} \times \sqrt{3}$ structures. 24 The latter structure has been shown to be more stable than the former due to quantum effects which choose this state from the multitude of classically degenerate states. 25. However, if one includes next nearest-neighbor interactions, the situation becomes more complicated. For second neighbor $\left(\mathrm{J}_{2}\right)$ and third neighbor interactions $\left(\mathrm{J}_{3}\right)$, the $\mathrm{q}=0$ state is preferred for $\mathrm{J}_{2}>\mathrm{J}_{3}$ and the $\mathrm{q}=\sqrt{3} \times \sqrt{3}$ for $\mathrm{J}_{2}<$ $\mathrm{J}_{3}$.26. The situation becomes even more elaborate if interplane interactions are considered as well. 27. More detailed studies are needed to clarify the nature of the spin interactions in $\mathrm{Li}_{2} \mathrm{Mn}_{2} \mathrm{O}_{4}$, such as been done recently for the jarosite $\mathrm{KCr}_{3} \mathrm{OD}_{6}\left(\mathrm{SO}_{4}\right)_{2}$ with inelastic neutron scattering experiments. 28

It is unclear why a three dimensional system such as $\mathrm{Li}_{2} \mathrm{Mn}_{2} \mathrm{O}_{4}$ would order with small regions of correlated $2 \mathrm{D}$ spins. Although these spin clusters have been observed in other spinels, as mentioned above, there is as of yet no explanation for why this would occur. The most intriguing work as of late is on the spinel $\mathrm{ZnCr}_{2} \mathrm{O}_{4}$, in which the authors, through neutron scattering experiments, demonstrate that hexagonal, 2D spin clusters play an important role in the low-temperature spin dynamics. 29] These hexagonal "protectorates" form independently of one another, and their excitations are the origin for the local zero energy modes in the pyrochlore lattice. It is suggested that the formation of spin clusters might be a central theme of self-organization in frustrated materials, which in many cases cannot have conventional
Néel ground states. Future work to measure the inelastic spectra of $\mathrm{Li}_{2} \mathrm{Mn}_{2} \mathrm{O}_{4}$ would be essential in elucidating the physics of these many-body systems.

C. R. Wiebe would like to acknowledge support from NSERC in the form of a PDF. The authors would like to thank the financial support of NSERC and CIAR. The authors are also grateful for the technical support of Bassam Hitti and Don Arseneau at TRIUMF.

* Current Address: Department of Physics, Brock University, St. Catharines, Ontario L2S 3A1, Canada; Electronic address: cwiebe@brocku.ca

[1] J. E. Greedan, Chem. Mater. 11, 37 (2001).

[2] A. P. Ramirez, Annu. Rev. Mater. Sci. 24, 453 (1994).

[3] S. Kondo, D. C. Johnson, C. A. Swenson, F. Borsa, A. V. Mahajan, L. L. Miller, T. Gu, A. I. Goldman, M. B. Maple, D. A. Gajewski, E. J. Freeman, N. R. Dilley, R. P. Dickey, J. Merrin, K. Kojima, G. M. Luke, Y. J. Uemura, O. Chmaissem, J. D. Jorgensen, Phys. Rev. Lett. 78, 3729 (1997).

[4] S. T. Bramwell and M. J. P. Gingras, Science 294, 1495 (2001).

[5] M. Hanawa, Y. Muraoka, T. Tayama, T. Sakakibara, J. Yamaura, Z. Hiroi, Phys. Rev. Lett. 87, 187001 (2001).

[6] P. W. Anderson, Phys. Rev. 102, 1008 (1956).

[7] J. E. Greedan, N. P. Raju, A. S. Wills, C. Morin and S. M. Shaw, Chem. Mater. 10, 3058 (1998).

[8] A. S. Wills, N. P. Raju and J. E. Greedan, Chem. Mater. 11, 1510 (1999).

[9] A. S. Wills, N. P. Raju, C. Moran and J. E. Greedan, Chem. Mater. 11, 1936, 1999.

[10] B. E. Warren, Phys. Rev. 59, 693, 1941.

[11] M. J. Ariza, D. J. Jones, J. Roziere, J. S. Lord, D. Ravot, J. Phys. Chem. B 107, 6003 (2003).

[12] A. Koda, R. Kadono, W. Higetomo, K. Ohishi, H. Ueda, C. Urano, S. Kondo, M. Nohara, H. Takagi, Phys. Rev. B 69, 012402 (2004).

[13] J. S. Gardner, S. R. Dunsiger, B. D. Gaulin, M. J. P. Gingras, J. E. Greedan, R. F. Kiefl, M. D. Lumsden, W. A. MacFarlane, N. P. Raju, J. E. Sonier, I. Swainson, Z. Tun, Phys. Rev. Lett. 82, 1012 (1999).

[14] S. R. Dunsiger, R. F. Kiefl, K. H. Chow, B. D. Gaulin, M. J. P. Gingras, J. E. Greedan, A. Keren, K. Kojima, G. M. Luke, W. A. MacFarlane, N. P. Raju, J. E. Sonier, Y. J. Uemura, W. D. Wu, Phys. Rev. B 54, 9019 (1996).

[15] M. T. Rovers, P. P. Kyriakou, H. A. Dabkowska, G. M. Luke, M. I. Larkin, and A. T. Savici, Phys. Rev. B. 66, 174434 (2002).

[16] Y. J. Uemura, A. Keren, K. Kojima, L. P. Le, G. M. Luke, W. D. Wu, Y. Ajiro, T. Asano, Y. Kuriyama, M. Mekata, H. Kikuchi, K. Kakurai, Phys. Rev. Lett. 73, 3306 (1994).

[17] A. T. Savici, Y. Fudamoto, I. M. Gat, T. Ito, M. I. Larken, Y. J. Uemura, G. M. Luke, K. M. Kojima, Y. S. Lee, M. A. Kastner, R. J. Birgineau, K. Yamada, Phys. Rev. B 66, 014524 (2002).

[18] A. P. Ramirez, E. S. Espinosa, A. S. Cooper, Phys. Rev. Lett. 45, 2505 (1992).

[19] W. Schiessl, W. Potzel, H. Karzel, M. Steiner, 
G. M. Kalvius, A. Martin, M. K. Krause, I. Halevy, J. Gal, W. Schafer, G. Will, M. Hillberg, R. Wappling, Phys. Rev. B 53, 9143 (1996).

[20] J. E. Greedan, C. R. Wiebe, A. S. Wills, and J. R. Stewart, Phys. Rev. B 65, 184424 (2002).

[21] J. D. M. Champion, A. S. Wills, T. Fennell, S. T. Bramwell, J. S. Gardner, M. A. Green, Phys. Rev. B 64, 140407 (2001).

[22] M. G. Townsend, G. Longworth and E. Roudant, Phys. Rev. B 33, 4919 (1986).

[23] A. S. Wills and A. Harrison, J. Chem. Soc. Faraday Trans. 92, 2161 (1996).

[24] A. Keren, K. Kojima, L. P. Le, G. M. Luke, W. D. Wu,
Y. J. Uemura, M. Takano, H. Dabkowska, M. J. P. Gingras, Phys. Rev. B 53, 6451 (1996).

[25] S. Sachdev, Phys. Rev. B 45, 12377 (1992).

[26] A. B. Harris, C. Kallin and A. J. Berlinsky, Phys. Rev. B 45, 2899 (1992).

[27] A. S. Wills, Phys. Rev. B 63, 064430 (2001).

[28] S.-H. Lee, C. Broholm, M. F. Collins, L. Heller, A. P. Ramirez, Ch. Kloc, E. Bucher, R. W. Erwin, N. Lacevic, Phys. Rev. B 56, 8091 (1997).

[29] S.-H. Lee, C. Broholm, W. Ratcliff, G. Gasparovic, Q. Huang, T. H. Kim, S. W. Cheong, Nature 418, 856 (2002). 